\title{
Muscle creatine kinase/SV40 hybrid promoter for muscle-targeted long-term transgene expression
}

\author{
FUMIHIKO TAKESHITA ${ }^{1}$, KEIKO TAKASE $^{1}$, MIYUKI TOZUKA ${ }^{1}$, SUKUMAR SAHA ${ }^{1}$, \\ KENJI OKUDA $^{1}$, NORIHISA ISHII ${ }^{2}$ and SHIN SASAKI ${ }^{1}$
}

${ }^{1}$ Department of Molecular Biodefense Research, Yokohama City University Graduate School of Medicine, Yokohama 236-0004; ${ }^{2}$ Department of Bioregulation, National Institute of Infectious Diseases, Tokyo 189-0002, Japan

Received September 25, 2006; Accepted November 2, 2006

\begin{abstract}
Gene therapy for congenital protein deficiencies requires lifelong expression of a deficient protein. Current gene therapy approaches preferentially employ the strong cytomegalovirus (CMV) promoter/enhancer or its derivative CAG promoter; however, these promoters provide only temporary transgene expression. To create a promoter that enables long-lasting expression in muscle, hybrid promoters were constructed by coupling the muscle creatine kinase (MCK) enhancer to various strong promoters for enhancement of tissue specificity and improved transcriptional activity. A hybrid promoter containing the MCK enhancer and the simian virus 40 promoter (MCK/SV40 promoter) yielded long-term (>6 months) expression of a human secretory alkaline phosphatase (huSEAP) reporter gene following electrotransfer of the plasmid into mice, whereas expression using a conventional CMV or CAG promoter faded away within a few weeks. To explore the mechanism behind the sustained expression obtained with the MCK/SV40 promoter, mice were immunized with a LacZ expression plasmid driven by MCK/SV40 or a conventional promoter. Minimal cellular and humoral responses to LacZ were observed in MCK/SV40 promotertreated animals, and mouse SEAP gene expression in vivo was successfully maintained by both the MCK/SV40 and conventional promoters. These results suggest that the lower immunogenicity of the MCK/SV40 promoter contributed to long-lasting gene expression in mice. Therefore, the MCK/ SV40 promoter may provide the basis for development of an effective transgene expression cassette for treatment of congenital protein deficiencies in which therapeutic proteins are recognized as foreign by the host immune system.
\end{abstract}

Correspondence to: Dr Fumihiko Takeshita, Department of Molecular Biodefense Research, Yokohama City University Graduate School of Medicine, 3-9 Fukuura, Kanazawa-ku, Yokohama 236-0004, Japan

E-mail: takesita@yokohama-cu.ac.jp

Key words: creatine kinase, SV40 hybrid promoter, transgene expression

\section{Introduction}

Skeletal muscle is easily accessible and readily expresses a transgene product; therefore, this muscle has been used as a platform for experimental gene therapy $(1,2)$. Muscle-targeted gene transfer has progressed rapidly from the pioneering demonstration of reporter gene expression by Wolff et al (3) to the production of various therapeutic proteins, including clotting factors (4), erythropoietin $(5,6)$, dystrophins (7), hormones (8), and enzymes responsible for lysosomal storage diseases (9). Such studies have shed new light on the treatment of genetic disorders; however, failure of persistence of gene expression hampers efforts towards the cure of these diseases, most of which require a lifelong supplement of the deficient protein.

The cytomegalovirus (CMV) immediate early gene promoter/enhancer (hereafter referred to as the CMV promoter) (10) or its derivative CAG promoter (11) has been used widely as a particularly powerful promoter for gene therapy, as well as for delivery of gene-mediated vaccines. Although the CMV promoter drives strong expression of the transgenes, it becomes silent within a few weeks of in vivo gene transfer $(12,13)$. In most cases, expression from the CMV promoter peaks 2 to 4 days after delivery of the transgene and declines to the background level within 4 weeks (14-16). This may be due to immunological elimination of cells bearing a foreign gene product $(14,17)$, since administration of neutralizing antibodies to transgene products (18-20) also results in such transgene silencing. Thus, the success of long-term gene therapy in part depends upon the ability of the promoter to avoid induction of immune responses to the transgene product.

Use of muscle-specific promoters is a promising strategy to suppress immunogenicity of transgenes $(21,22)$, since such tissue-specific promoters are inert in antigen presenting cells (APCs), whereas ubiquitous promoters such as CMV and CAG are active in these cells (23). However, a primary drawback of tissue-specific promoters is their relatively low potential for transcriptional activity compared with ubiquitous promoters. To improve the performance of muscle-specific promoters while retaining tissue specificity, we have constructed hybrid promoters by coupling the muscle creatine kinase (MCK) enhancer to various strong promoters. The combination of the MCK enhancer and the simian virus 40 promoter (MCK/SV40 
promoter) achieved long-term transgene expression following intramuscular electrotransfer, while inducing only marginal levels of cellular and humoral immune responses to transgene products. Our results suggest that the MCK/SV40 promoter is a promising substitute for conventional CMV-based promoters when long-term transgene expression in the muscle is required.

\section{Materials and methods}

Plasmid construction and preparation. Plasmid construction was based on the plasmid pGA (24), which consists of the $\mathrm{CMV}$ promoter plus intron $\mathrm{A}$, bovine growth hormone terminator, $\mathrm{Kan}^{\mathrm{r}}$, and pUC ori. Schematic diagrams of the promoters characterized in this study are shown in Fig. 1. The mouse MCK promoter (nucleotides -1256 to +7 ; GeneBank accession no. AF188002) was PCR-amplified from genomic DNA from RAW264.7 cells using the primer pair 5'-GAC TAG TCC ACT ACG GGT CTA GGC TGC CC-3' and 5'-CCC AAG CTT GGG GGC AGC CCC TGT GCC CCT GGG-3'. The CMV promoter plus intron A in pGA was released by digestion with SpeI and HindIII and replaced with the SpeI-HindIII MCK promoter amplicon. Similarly, the SpeI-EcoRI CAG promoter fragment was excised from the pCAGGS vector (11) and substituted for the CMV promoter plus intron A, resulting in the pGA-CAG vector. To create a series of hybrid promoters, the MCK promoter containing an enhancer region (25) and another promoter were coupled by ligation. The SV40 promoter was excised from the pGL3control vector (Promega, Madison, WI) with SmaI and HindIII or NheI and HindIII. The pGA-MCK vector was digested with SmaI and HindIII or NheI and HindIII, and then ligated with the SmaI-HindIII or NheI-HindIII SV40 promoter fragment, respectively. The ligation products contained a hybrid promoter consisting of the MCK enhancer and SV40 promoter and were designated as pGA-MCK/SV40 (L) and pGA-MCK/SV40 (S), respectively. The CMV enhancer in pGA-CAG was replaced with the SpeI-SmaI MCK fragment carrying an enhancer region to create the plasmid pGA-MCK/ß-actin, which contains a hybrid promoter comprising the MCK enhancer and chicken ß-actin promoter. Similarly, the CMV enhancer in the pGA vector was replaced with the MCK enhancer to generate pGA$\mathrm{MCK} / \mathrm{CMV}$, which contains a hybrid promoter consisting of the MCK enhancer and CMV promoter. The reporter genes, firefly luciferase (luc) from pGL3-control, ß-galactosidase (LacZ) from $\mathrm{pSV}$ - $\beta$-galactosidase (both from Promega), human secretory alkaline phosphatase (huSEAP) from pSEAP2-Basic (BD-Clontech, Palo Alto, CA), and mouse SEAP, which was cloned as described previously (26), were subcloned into vectors containing each specific promoter. All plasmids were produced by growing transformed E. coli $\mathrm{DH} 5 \alpha$ in LB media and then purified on Qiagen DNA purification columns (Qiagen, Hilden, Germany).

Transfection and luciferase assay. Human embryonic kidney 293 cells or mouse myoblast C2C12 cells were cultured in high-glucose DMEM supplemented with $10 \%$ FCS. The cells were seeded in 96-well tissue culture plates and grown until $50-70 \%$ confluency. They were then transiently transfected with each luciferase reporter plasmid using FuGene 6 transfection reagent (Roche, Mannheim, Germany). Two days after

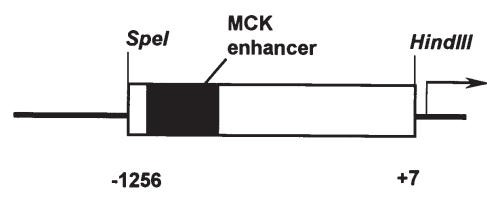

MCK/SV40 (S)

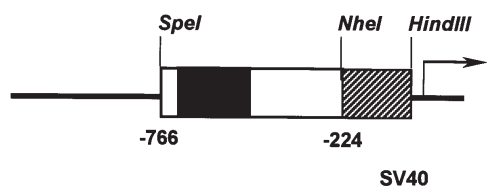

promoter
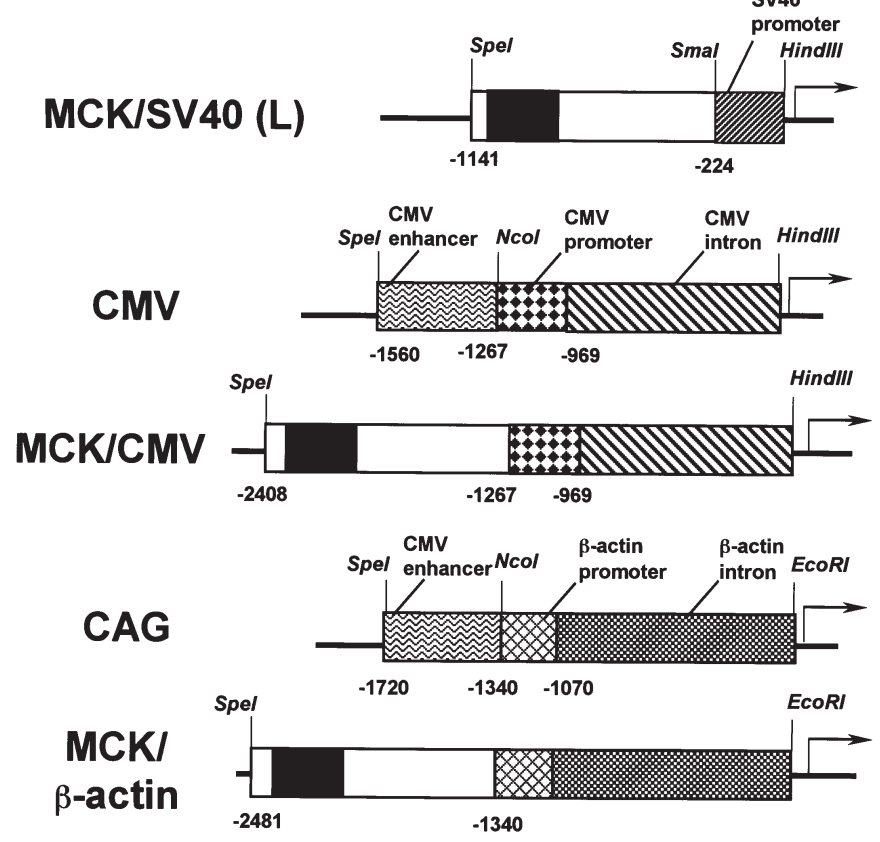

Figure 1. Schematic presentation of promoters that were constructed and evaluated in the study. MCK, muscle creatine kinase; SV40, simian virus 40; CMV, cytomegalovirus immediate early gene enhancer/promoter plus intron A; CAG, cytomegalovirus enhancer and chicken $ß$-actin hybrid promoter.

transfection, the luminescence signal was measured using a microplate luminometer (Veritas, Turner Biosystems, Sunnyvale, CA) with a Dual-Glo luciferase assay kit (Promega) according to the manufacturer's instructions. phRL-TK expressing Renilla luciferase (Promega) was used as a secondary reporter vector for normalization of firefly reporter activity.

Animal treatment and measurement of plasma SEAP activity. Female BALB/c mice were purchased from Japan SLC Inc. (Shizuoka, Japan) and housed in the animal facility of Yokohama City University Graduate School of Medicine. DNA injection, electroporation, and retro-orbital puncture for blood sampling were all performed under anaesthesia produced by subcutaneous injection of a 4:1 mixture of ketamine and xylazine. Animal experiments were conducted under the approval of the institutional laboratory animal care and use committee. For electroporation, the quadriceps femoris muscles were exposed and injected with the indicated dose of plasmid in saline with a 30-gauge needle inserted 2-3 mm deep, and then the entire muscle bundle was pinched by a pair of disktype platinum electrodes (diameter: $10 \mathrm{~mm}$, separation: $\sim 5 \mathrm{~mm}$; CUY650-10, Nepa Gene, Chiba, Japan). Six electrical pulses were applied at $30 \mathrm{~V}$ using a square wave electroporator 


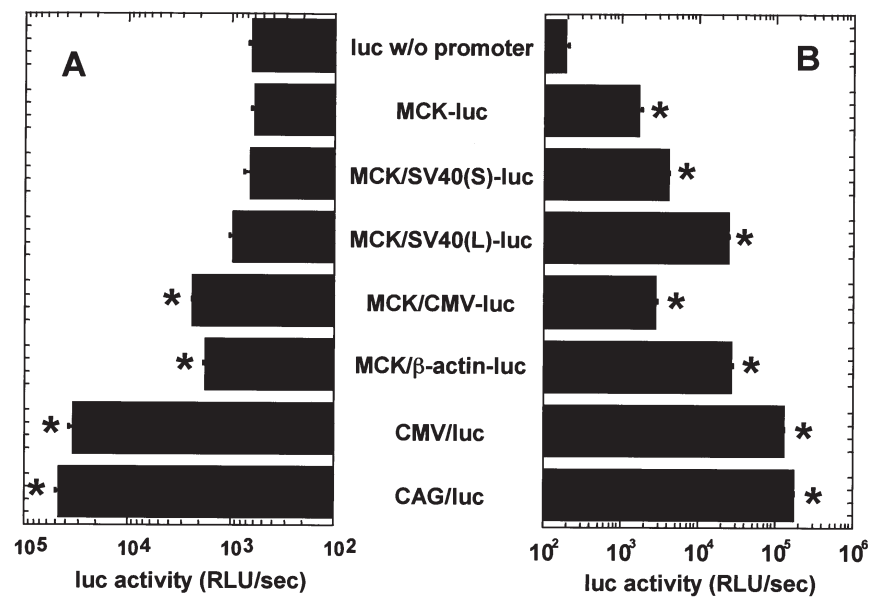

Figure 2. In vitro characterization of hybrid and conventional promoters. Luciferase activities were measured in human embryonic kidney 293 cells (A) and mouse myoblast $\mathrm{C} 2 \mathrm{C} 12$ cells (B) transiently transfected with the indicated luciferase expression plasmids. The cells were transfected with $50 \mathrm{ng} /$ well of the indicated plasmid, and the luciferase activity was measured 2 days after transfection. The data shown are averages of six wells, and error bars indicate the standard errors. The experiments were repeated twice with similar results. ${ }^{*} \mathrm{p}<0.05$.

CUY21EDIT (Nepa Gene) with a pulse duration of $50 \mathrm{~ms}$, a pulse interval of $100 \mathrm{~ms}$, and reversal of polarity after three pulses. This procedure was completed by closure of the incision with surgical wound clips. For hydrodynamics-based transfection to the liver (27), $10 \mu \mathrm{g}$ of each plasmid was formulated with a polymer (Trans-IT in vivo transfection reagent, Mirus, Madison, WI) and dissolved with Ringer's solution. The DNA/polymer complex solution $(2.5 \mathrm{ml})$ was injected into the tail vein of each mouse over 6-8 sec using a 27 -gauge needle. Scheduled blood samples were taken by retro-orbital puncture and stored at $-20^{\circ} \mathrm{C}$. Plasma SEAP activity was measured using a SEAP reporter gene chemiluminescent assay kit (Roche) and a microplate luminescence reader according to the manufacturer's instructions.

CTL assay. Splenocytes were harvested 2 weeks after the booster immunization. The CTL isolates (effector cells) were prepared after incubation with $1 \mu \mathrm{g} / \mathrm{ml}$ of $\mathrm{H}-2^{\mathrm{d}}$-restricted LacZ class I peptide (TPHPARIGL) and $20 \mathrm{U} / \mathrm{ml}$ of IL-2 (Sigma) for 4 days at $37^{\circ} \mathrm{C}$. The target cells were mouse mastocytoma P815 cells $\left(\mathrm{H}-2 \mathrm{~K}^{\mathrm{d}}\right)$ pulsed with $1 \mu \mathrm{g} / \mathrm{ml}$ of $\mathrm{H}-2^{\mathrm{d}}$-restricted LacZ class I peptide (experimental target) or $\mathrm{H}-2^{\mathrm{b}}$-restricted LacZ class I peptide (DAPIYTNV, sham target). The target cells $\left(1 \times 10^{4}\right)$ were co-cultured with the effector cells for $4 \mathrm{~h}$. The amount of lactate dehydrogenase (LDH) released from the target cells was measured using a Cytotox 96 system (Promega) and \% specific lysis was calculated according to the manufacturer's protocol.

ELISA. The serum Ab titer was measured by ELISA, as described elsewhere (24).

Statistics. Statistical analysis was conducted with Student's t-test using StatView J-4.02 software (Abacus Concepts, Berkeley, CA), and significance was defined as $\mathrm{p}<0.05$.

\section{Results}

In vitro characterization of the hybrid promoters. To optimize the promoter for muscle-targeting gene therapy vectors, various hybrid promoters comprising the MCK enhancer and the SV40, CMV or CAG ( $ß$-actin) promoter were constructed as shown in Fig. 1. Promoter activity was tested in vitro using HEK 293 (embryonal kidney) cells and C2C12 (myoblast) cells (Fig. 2). As shown in Fig. 2A, the promoter activities of MCK and MCK/SV40 (S) were similar to that of the control (luc w/o promoter) in HEK 293 cells, whereas conventional
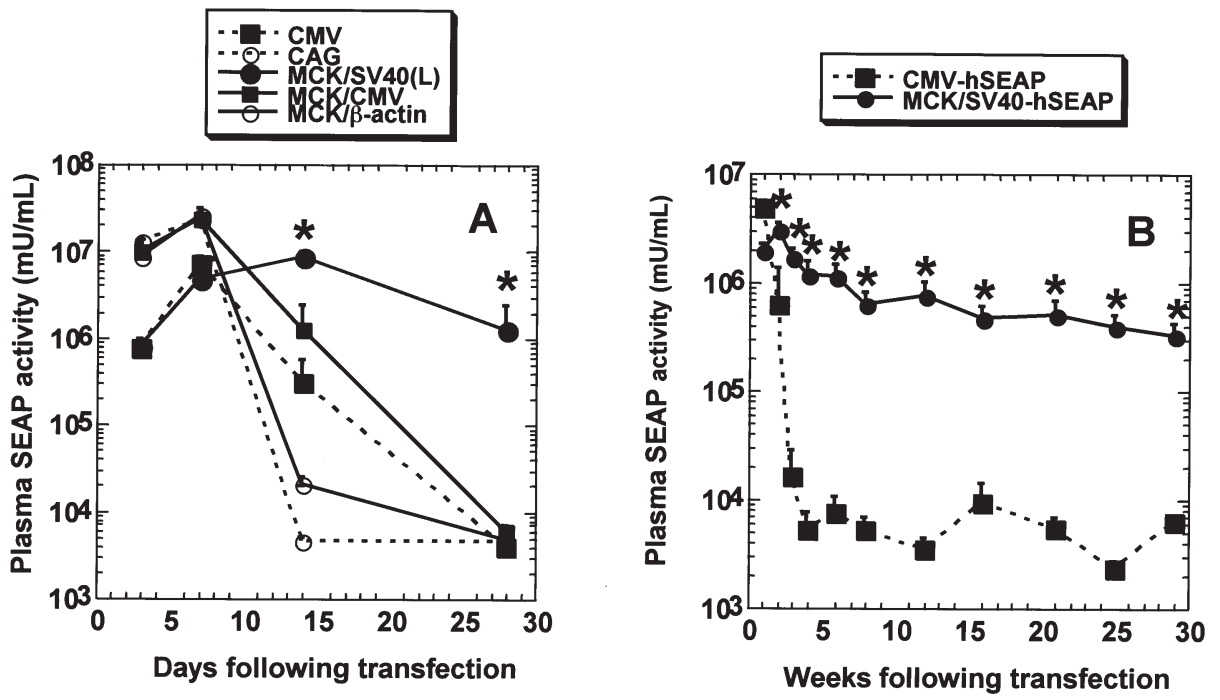

Figure 3. In vivo characterization of hybrid and conventional promoters (A) and long-term follow-up of transgene expression by the MCK/SV40 (L) promoter (B). Plasma SEAP activities were measured in mice transfected with the human SEAP expression plasmid under the transcriptional control of the indicated promoter. The mice (8 in each group) received intramuscular electrotransfer of $50 \mu \mathrm{g}$ of plasmid on day 0 , and plasma SEAP activity was then monitored continuously. The data shown are averages of eight samples, and error bars show standard errors. An independent experiment gave similar results for each promoter. ${ }^{*} \mathrm{p}<0.05$. 


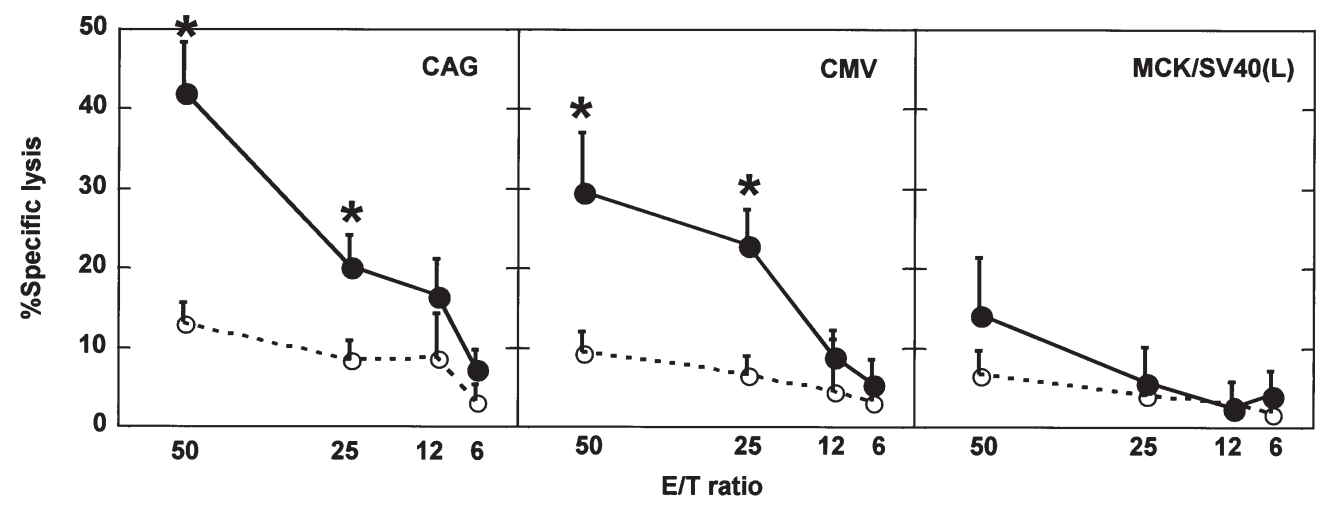

Figure 4. LacZ-specific cytolytic response of splenocytes harvested from animals that underwent electrotransfer of the indicated promoter-driven LacZ expression plasmid. Mice were immunized at 0 and 4 weeks, and splenic mononuclear cells were harvested at 6 weeks and cultured with haplotype-matched $\left(\mathrm{H}-2^{\mathrm{d}}\right)$ LacZ class I peptide for 4 days. Mouse mastocytoma P815 cells $\left(\mathrm{H}-2 \mathrm{~K}^{\mathrm{d}}\right)$ were pulsed with $1 \mu \mathrm{g} / \mathrm{ml}$ of haplotype-matched (H-2 ; filled circles) or haplotype-unmatched (H-2 $2^{\text {b }}$ open circles) class I peptide for $1 \mathrm{~h}$ and used as target cells. The effector and target cells were mixed at E:T ratios of 6-50 and cultured for $4 \mathrm{~h}$, and the released LDH activity was measured by colorimetric assay. The graph shows the mean \pm SEM of the $\%$ specific lysis of five animals in each group. Similar results were obtained in a repeated experiment for each promoter. ${ }^{*} \mathrm{p}<0.05$.

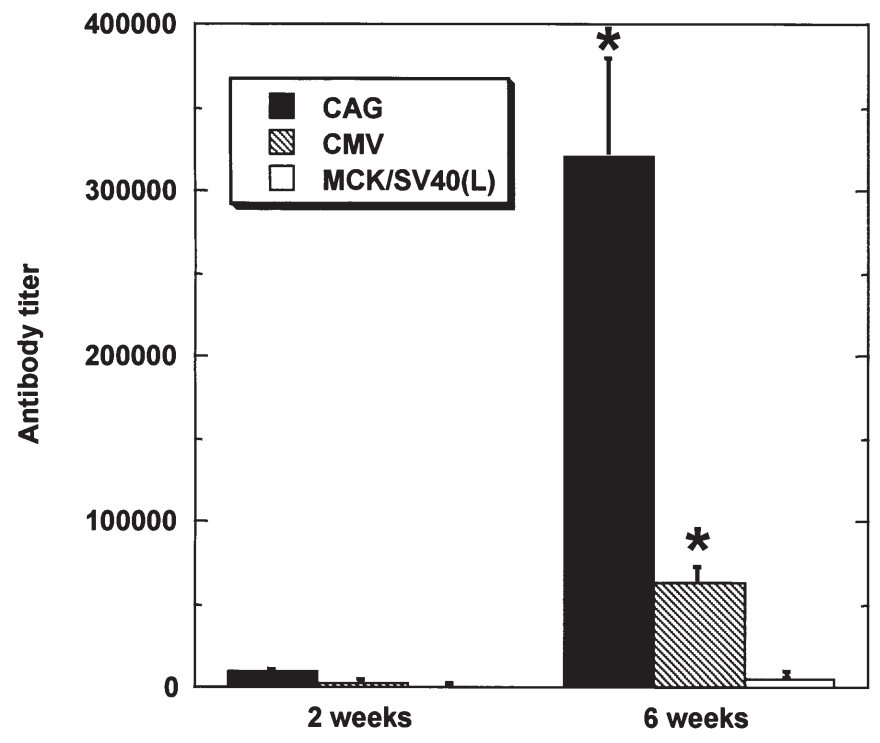

Figure 5. LacZ-specific antibody titer of sera from animals that underwent electrotransfer of the indicated promoter-driven LacZ expression plasmid. Mice were immunized at 0 and 4 weeks, and samples of immune sera were collected at 2 and 6 weeks for antibody titration by ELISA. The graph shows the mean \pm SEM of antibody titer of five animals in each group. Similar results were obtained in a repeated experiment for each promoter. ${ }^{*} \mathrm{p}<0.05$.

CMV and CAG promoters exhibited robust activities. Among the hybrid promoters, the MCK/CMV promoter showed the best performance, reaching approximately one-tenth of the expression level obtained under control of the CMV or CAG promoter. In contrast, $\mathrm{MCK}$ and the hybrid promoters yielded substantial reporter gene expression in $\mathrm{C} 2 \mathrm{C} 12$ cells (Fig. 2B) and the MCK/CMV (L) promoter showed the best performance among the hybrids, suggesting that the activities of the MCK promoter and derivatives are higher in myoblasts than in non-muscle cells. Based upon the good performance in C2C12 cells, MCK/SV40 (L), MCK/CMV and MCK/B-actin promoters were used for in vivo characterization.
In vivo characterization of hybrid promoters. We next examined whether the hybrid promoters increased transgene expression in mouse muscle, using a human placental secretory alkaline phosphatase (huSEAP) reporter gene. SEAP activity can be monitored in a plasma-based assay that allows longterm surveillance of the gene expression profile without sacrificing the animals. We chose the human gene, and not mouse SEAP, due to the immunogenicity of human SEAP in mice $(28,29)$; this protocol mimics the conditions in gene therapy for congenital protein deficiencies, since proteins responsible for congenital deficiencies are often recognized as foreign by the immune system (30-32). SEAP expression profiles in mice that received intramuscular electrotransfer of a human SEAP expression plasmid are shown in Fig. 3. It is clear that only the MCK/SV40 (L) promoter maintained SEAP activity after 4 weeks, whereas expression with other promoters declined rapidly to the background level. Prolonged monitoring was used to test the long-term sustainability of huSEAP expression under control of the MCK/SV40 (L) promoter, as shown in Fig. 3B. The MCK/SV40 (L) promoter was able to sustain SEAP expression for 29 weeks, whereas expression with the control CMV promoter decreased to below the background level within 4 weeks. These findings suggest that the MCK/SV40 (L) promoter is a promising substitute for the conventional CMV promoter when longterm expression is required for immunogenic transgene products.

Immunogenicity of the MCK/SV4O (L) promoter. Immunemediated elimination of cells bearing a transgene product $(14,17)$ and neutralizing antibodies to transgene products (18-20) might account for the transient expression observed with conventional $\mathrm{CMV}$ and $\mathrm{CAG}$ promoters. Thus, the above results suggest that the MCK/SV40 (L) promoter has weak immunogenicity, which allows long-term transgene expression. To assess the immunogenic properties of the MCK/SV40 (L) promoter, LacZ expression plasmids driven by the MCK/SV40 (L), CAG, and CMV promoters were constructed and administered to mice. The LacZ-specific 

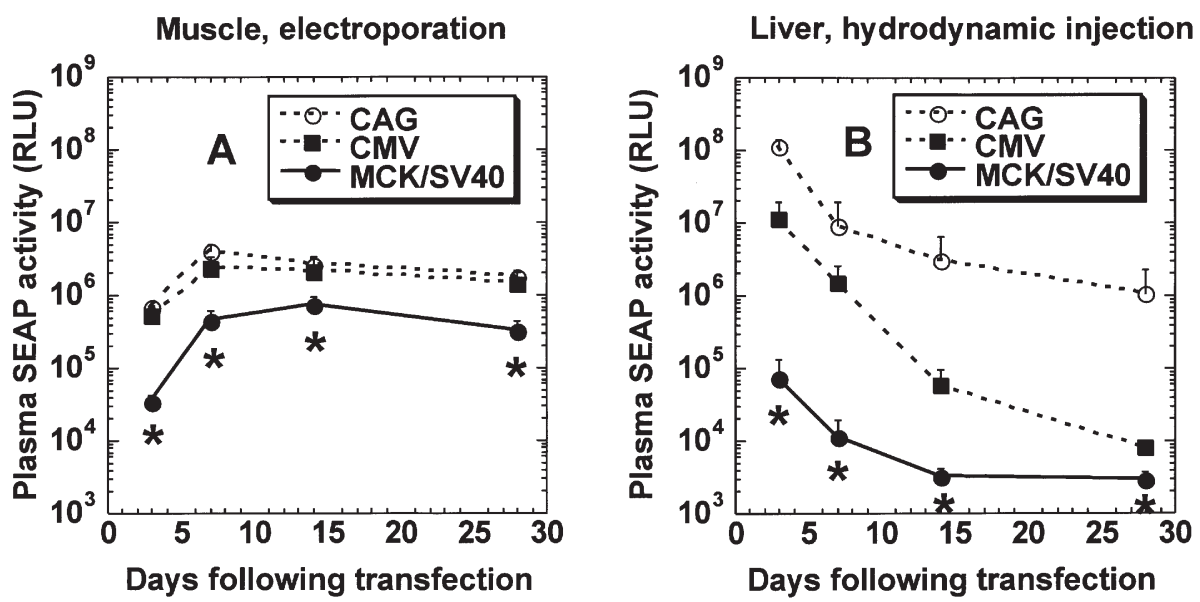

Figure 6. Mouse SEAP expression profiles following intramuscular electrotransfer (left) and hydrodynamic tail vein injection (right) of the indicated promoter-driven mouse SEAP expression plasmid. The mice (8 in each group) received intramuscular electrotransfer or hydrodynamic tail vein injection of $100 \mu \mathrm{g}$ of each plasmid on day 0, and plasma SEAP activity was then monitored continuously. The data shown are averages of eight samples, and error bars show standard errors. An independent experiment gave similar results for each promoter. ${ }^{*} \mathrm{p}<0.05$

cytotoxic T-lymphocyte (CTL) activity of splenocytes from mice immunized with each plasmid is shown in Fig. 4. It is noteworthy that both the CAG and CMV promoters elicited considerable lytic activity, whereas the activity elicited by the MCK/SV40 (L) promoter remained at a background level. Similar results were observed when humoral immune responses were examined (Fig. 5). Immunization with a plasmid containing CAG or CMV promoter resulted in substantial antibody production after 6 weeks, with the CAG promoter producing more antibodies than the CMV promoter, as reported previously (33). The MCK/SV40 (L) promoter generated a minimal antibody response after 6 weeks; overall, the cellular and humoral immune responses in the presence of the MCK/SV40 (L) promoter suggest that this promoter has minimal immunogenicity.

Expression levels of non-immunogenic transgene products under the control of MCK/SV4O (L) promoter. The above results indicate that the MCK/SV40 (L) promoter enables long-lasting expression of huSEAP and avoids elicitation of immune responses to lacZ protein. To further characterize the $\mathrm{MCK} / \mathrm{SV} 40$ (L) promoter, the in vivo transgene expression profile of the mouse SEAP (mSEAP) reporter gene was examined, since this gene is non-immunogenic to mice. mSEAP expression profiles with the CMV, CAG, and MCK/ SV40(L) promoters are shown in Fig. 6A. In contrast to the results for huSEAP, sustained expression of mSEAP was observed with the CMV and CAG promoters. Mouse SEAP expression with the MCK/SV40 (L) promoter was also sustainable, but the expression level was only about one-tenth that with the CMV or CAG promoter. Considering both the human and mouse SEAP expression profiles, it was concluded that intramuscular transfection of the MCK/SV40 (L) promoter allowed long-term expression of an immunogenic transgene, but that the actual promoter activity in the muscle was moderate. In contrast, conventional CMV and CAG promoters are much stronger in the muscle, but could not sustain expression of an immunogenic transgene product after intramuscular electrotransfer. To assess the tissue specificity of
MCK/SV40 (L) promoter activity, mSEAP-expressing plasmids driven by a CAG, CMV or MCK/SV40 (L) promoter were transfected into the liver, using a conventional hydrodynamic method. As shown in Fig. 6B, SEAP activity with the MCK/SV40 (L) promoter was about one-hundredth of that with the conventional promoters, suggesting muscle specificity of MCK/SV40 (L) promoter activity.

\section{Discussion}

To examine long-term transgene expression, a hybrid MCK/ SV40 (L) promoter was generated by coupling the muscle creatine kinase enhancer to the SV40 promoter. The levels of immunogenic and non-immunogenic transgene products under control of the novel promoter were compared with those produced by conventional ubiquitous $\mathrm{CAG}$ and $\mathrm{CMV}$ promoters after intramuscular electrotransfer in mice. A key finding in the study is that the MCK/SV40 (L) promoter achieved long-term expression of an immunogenic reporter gene with minimal induction of cellular and humoral immune responses to the transgene product.

Muscle has been a major target in attempts to seek effective gene therapy using plasmid vectors, because it is easily accessible and efficiently secretes the transgene product into the systemic circulation $(1,2)$. Thus, improvement of gene expression in muscle is of importance for the progress of gene therapy for congenital disorders that can be treated with a therapeutic gene product in the circulation, such as hemophilia (34) and lysosomal storage diseases (9). Previous studies have shown that intramuscular plasmid injection results in production of therapeutic protein in treatment of each of these diseases $(16,35)$. However, the lack of persistent gene expression hampers efforts to supply therapeutic levels of gene products. In this regard, the MCK/SV40 (L) promoter might be suited for gene therapy of these and other disorders that can be treated by supplying a deficient protein in the systemic circulation.

Another concern in gene therapy for congenital disorders is elicitation of immune responses against the deficient 
protein, since the patient's immune system might recognize a previously unexpressed protein as foreign. In fact, production of antibodies against deficient proteins has been reported in patients undergoing enzyme replacement therapy for congenital deficiencies such as hemophilia A and B (32), adenosine deaminase deficiency (36), mucopolysaccharidosis type I (30), Gaucher disease (37), Fabry disease (31), and Pompe disease (38). In these patients, the antibodies may neutralize the bioactivity of the therapeutic protein; moreover, administration of the vector encoding the therapeutic protein might augment the immune response to a transgene, as seen in prime-boost immunization of DNA vaccines. Two pathways have been proposed in DNA-raised specific immune responses following intramuscular injection with DNA vaccines. APCs recruited to the muscles may take up DNA and express and process the encoding Ag for direct presentation to cognate T- and Blymphocytes (direct-priming). Alternatively, Ag proteins expressed by muscle cells may be taken up and processed by APCs and subsequently presented to Ag-specific lymphocytes (cross-priming). Moreover, reports from other groups and our previous studies have shown that direct transfection of DNA vaccines into APCs by intramuscular electroporation is responsible for subsequent immunogenicity (39-41). Such evidence strongly suggests that the minimal immunogenicity of the MCK/SV40 promoter is due to its weak activity, especially in transfected APCs.

The long-lasting expression of the huSEAP gene under the control of the MCK/SV40 (L) promoter suggests that this promoter has favorable characteristics for gene therapy targeted to congenital protein deficiencies. It will be of interest to examine the expression profiles of therapeutic genes, rather than reporter genes, using the MCK/SV40 (L) promoter to determine whether the promoter is functional in a gene therapy model. With allogeneic mSEAP as a reporter gene, SEAP expression persisted for at least 4 weeks with all tested promoters. This is in marked contrast to the rapid decrease in SEAP activity observed in huSEAP-treated animals. The difference in expression of human and mouse SEAP indicates that huSEAP clearance occurs through an immune-mediated mechanism, which is consistent with previous observations showing that the plasma huSEAP level is in inverse proportion to the anti-huSEAP antibody titer $(15,29,42)$. As noted, the strongest SEAP expression was attained with conventional promoters, and not with the MCK/SV40 (L) promoter, which might be a reflection of the net promoter activity for a nonimmunogenic reporter gene. This suggests that the MCK/ SV40 (L) promoter is unlikely to improve transgene expression for a non-immunogenic gene. However, studies of gene transfer of allogeneic factor VIII $(20,43,44)$, IX $(45,46)$, and erythropoietin $(19,47)$ into animals indicate the risk of eliciting immune responses against a transgene, even if it originates from the same species. Thus, we believe that the MCK/SV40 (L) promoter might confer considerable benefit in gene therapy for congenital protein deficiencies in which the therapeutic gene is recognized as foreign by the immune system.

In conclusion, the novel promoter created in the current study, the MCK/SV40 (L) promoter, might be a promising substitute for conventional $\mathrm{CMV}$ and $\mathrm{CAG}$ promoters when persistent expression is required for a transgene that triggers immune responses. The current results provide the basis for further advances in muscle-targeted gene therapy for congenital protein deficiencies, and studies are now necessary to determine whether this novel promoter can achieve sustainable expression of genes associated with congenital protein deficiencies.

\section{Acknowledgements}

We would like to thank A. de la Fuente for administrative assistance and A. Sakata and T. Matsuda for technical assistance. This work was supported by a Grant-In-Aid from the National Institute of Biomedical Innovation, a Grant-In-Aid for the Advancement of Medical Science from the Yokohama Foundation for Medical Science Promotion, a Precedent Research Grant for Frontline Medical Science from Yokohama City University, and a Grant-In-Aid for Scientific Research from the Ministry of Education, Culture, Sports, Science, and Technology of Japan.

\section{References}

1. Lu QL, Bou-Gharios G and Partridge TA: Non-viral gene delivery in skeletal muscle: a protein factory. Gene Ther 10 : 131-142, 2003.

2. Herweijer H and Wolff JA: Progress and prospects: naked DNA gene transfer and therapy. Gene Ther 10: 453-458, 2003.

3. Wolff JA, Malone RW, Williams P, Chong W, Acsadi G, Jani A and Felgner PL: Direct gene transfer into mouse muscle in vivo. Science 247: 1465-1468, 1990.

4. Chuah MK, Collen D and Van den Driessche T: Gene therapy for hemophilia. J Gene Med 3: 3-20, 2001.

5. Bohl D and Heard JM: Delivering erythropoietin through genetically engineered cells. J Am Soc Nephrol 11: S159-S162, 2000

6. Kreiss P, Bettan M, Crouzet J and Scherman D: Erythropoietin secretion and physiological effect in mouse after intramuscular plasmid DNA electrotransfer. J Gene Med 1: 245-250, 1999.

7. Chamberlain JS: Gene therapy of muscular dystrophy. Hum Mol Genet 11: 2355-2362, 2002.

8. MacColl GS, Novo FJ, Marshall NJ, Waters M, Goldspink G and Bouloux PM: Optimisation of growth hormone production by muscle cells using plasmid DNA. J Endocrinol 165: 329-336, 2000.

9. Cheng SH and Smith AE: Gene therapy progress and prospects: gene therapy of lysosomal storage disorders. Gene Ther 10: 1275-1281, 2003.

10. Boshart M, Weber F, Jahn G, Dorsch-Hasler K, Fleckenstein B and Schaffner W: A very strong enhancer is located upstream of an immediate early gene of human cytomegalovirus. Cell 41: $521-530,1985$.

11. Miyazaki J, Takaki S, Araki K, Tashiro F, Tominaga A, Takatsu K and Yamamura K: Expression vector system based on the chicken beta-actin promoter directs efficient production of interleukin-5. Gene 79: 269-277, 1989.

12. Bloquel C, Fabre E, Bureau MF and Scherman D: Plasmid DNA electrotransfer for intracellular and secreted proteins expression: new methodological developments and applications. J Gene Med 6: S11-S23, 2004.

13. Smith LC and Nordstrom JL: Advances in plasmid gene delivery and expression in skeletal muscle. Curr Opin Mol Ther 2: $150-154,2000$.

14. Payette PJ, Weeratna RD, McCluskie MJ and Davis HL: Immune-mediated destruction of transfected myocytes following DNA vaccination occurs via multiple mechanisms. Gene Ther 8: 1395-1400, 2001.

15. Gronevik E, von Steyern FV, Kalhovde JM, Tjelle TE and Mathiesen I: Gene expression and immune response kinetics using electroporation-mediated DNA delivery to muscle. J Gene Med 7: 218-227, 2005.

16. Bettan M, Emmanuel F, Darteil R, Caillaud JM, Soubrier F, Delaere P, Branelec D, Mahfoudi A, Duverger N and Scherman D: High-level protein secretion into blood circulation after electric pulse-mediated gene transfer into skeletal muscle. Mol Ther 2: 204-210, 2000. 
17. Ghazizadeh S, Kalish RS and Taichman LB: Immune-mediated loss of transgene expression in skin: implications for cutaneous gene therapy. Mol Ther 7: 296-303, 2003.

18. Bettan M, Darteil R and Scherman D: Secreted human placental alkaline phosphatase as a reporter gene for in vivo gene transfer. Anal Biochem 271: 187-189, 1999.

19. Chenuaud P, Larcher T, Rabinowitz JE, Provost N, Cherel Y, Casadevall N, Samulski RJ and Moullier P: Autoimmune anemia in macaques following erythropoietin gene therapy. Blood 103: 3303-3304, 2004.

20. Wu H, Reding M, Qian J, Okita DK, Parker E, Lollar P, Hoyer LW and Conti-Fine BM: Mechanism of the immune response to human factor VIII in murine hemophilia $\mathrm{A}$. Thromb Haemost 85: 125-133, 2001.

21. Dunant P, Larochelle N, Thirion C, Stucka R, Ursu D, Petrof BJ, Wolf $\mathrm{E}$ and Lochmuller $\mathrm{H}$ : Expression of dystrophin driven by the 1.35-kb MCK promoter ameliorates muscular dystrophy in fast, but not in slow muscles of transgenic mdx mice. Mol Ther 8: 80-89, 2003.

22. Yoshimura M, Sakamoto M, Ikemoto M, Mochizuki Y, Yuasa K, Miyagoe-Suzuki Y and Takeda S: AAV vector-mediated microdystrophin expression in a relatively small percentage of $\mathrm{mdx}$ myofibers improved the mdx phenotype. Mol Ther 10: 821-828, 2004.

23. Weeratna RD, Wu T, Efler SM, Zhang L and Davis HL: Designing gene therapy vectors: avoiding immune responses by using tissue-specific promoters. Gene Ther 8: 1872-1878, 2001.

24. Sasaki S, Amara RR, Oran AE, Smith JM and Robinson HL: Apoptosis-mediated enhancement of DNA-raised immune responses by mutant caspases. Nat Biotechnol 19: 543-547, 2001.

25. Donoviel DB, Shield MA, Buskin JN, Haugen HS, Clegg CH and Hauschka SD: Analysis of muscle creatine kinase gene regulatory elements in skeletal and cardiac muscles of transgenic mice. Mol Cell Biol 16: 1649-1658, 1996.

26. Wang M, Orsini C, Casanova D, Millan JL, Mahfoudi A and Thuillier V: MUSEAP, a novel reporter gene for the study of long-term gene expression in immunocompetent mice. Gene 279: 99-108, 2001.

27. Liu F, Song Y and Liu D: Hydrodynamics-based transfection in animals by systemic administration of plasmid DNA. Gene Ther 6: 1258-1266, 1999.

28. Gronevik E, Mathiesen I and Lomo T: Early events of electroporation-mediated intramuscular DNA vaccination potentiate Th1-directed immune responses. J Gene Med 7: 1246-1254, 2005.

29. Chastain M, Simon AJ, Soper KA, Holder DJ, Montgomery DL, Sagar SL, Casimiro DR and Middaugh CR: Antigen levels and antibody titers after DNA vaccination. J Pharm Sci 90: 474-484, 2001.

30. Kakkis ED, Muenzer J, Tiller GE, Waber L, Belmont J, Passage M, Izykowski B, Phillips J, Doroshow R, Walot I, Hoft R and Neufeld EF: Enzyme-replacement therapy in mucopolysaccharidosis I. N Engl J Med 344: 182-188, 2001.

31. Eng CM, Banikazemi M, Gordon RE, Goldman M, Phelps R, Kim L, Gass A, Winston J, Dikman S, Fallon JT, Broide S, Stacy CB, Mehta D, Parsons R, Norton K, O'Callagham M and Desnick RJ: A phase 1/2 clinical trial of enzyme replacement in Fabry disease: pharmacokinetic, substrate clearance, and safety studies. Am J Hum Genet 68: 711-722, 2001.

32. Scharrer I, Bray GL and Neutzling O: Incidence of inhibitors in haemophilia A patients - a review of recent studies of recombinant and plasma-derived factor VIII concentrates. Haemophilia 5: 145-154, 1999.
33. Garg S, Oran AE, Hon H and Jacob J: The hybrid cytomegalovirus enhancer/chicken beta-actin promoter along with woodchuck hepatitis virus posttranscriptional regulatory element enhances the protective efficacy of DNA vaccines. J Immunol 173: 550-558, 2004.

34. Walsh C: Gene therapy for hemophilia: are viral vectors really feasible? J Thromb Haemost 1: 218-219, 2003.

35. Yew NS, Przybylska M, Ziegler RJ, Liu D and Cheng SH: High and sustained transgene expression in vivo from plasmid vectors containing a hybrid ubiquitin promoter. Mol Ther 4: 75-82, 2001.

36. Chaffee S, Mary A, Stiehm ER, Girault D, Fischer A and Hershfield MS: IgG antibody response to polyethylene glycolmodified adenosine deaminase in patients with adenosine deaminase deficiency. J Clin Invest 89: 1643-1651, 1992.

37. Richards SM, Olson TA and McPherson JM: Antibody response in patients with Gaucher disease after repeated infusion with macrophage-targeted glucocerebrosidase. Blood 82: 1402-1409, 1993.

38. Chen YT and Amalfitano A: Towards a molecular therapy for glycogen storage disease type II (Pompe disease). Mol Med Today 6: 245-251, 2000.

39. Torres CA, Iwasaki A, Barber BH and Robinson HL: Differential dependence on target site tissue for gene gun and intramuscular DNA immunizations. J Immunol 158: 4529-4532, 1997.

40. Takeshita F, Tanaka T, Matsuda T, Tozuka M, Kobiyama K, Saha S, Matsui K, Ishii KJ, Coban C, Akira S, Ishii N, Suzuki K, Klinman DM, Okuda K and Sasaki S: Toll-like receptor adaptor molecules enhance DNA-raised adaptive immune responses against influenza and tumors through activation of innate immunity. J Virol 80: 6218-6224, 2006.

41. Sasaki S, Smith JM, Takase K, Okuda K, Ishii N and Takeshita F: Activator protein 1-mediated transcriptional regulation strategy sustains long-term expression of a xenogeneic gene product in vivo: an implication for gene therapy targeting congenital protein deficiencies. Int J Mol Med 18: 289-297, 2006.

42. Maelandsmo GM, Ross PJ, Pavliv M, Meulenbroek RA, Evelegh C, Muruve DA, Graham FL and Parks RJ: Use of a murine secreted alkaline phosphatase as a non-immunogenic reporter gene in mice. J Gene Med 7: 307-315, 2005.

43. Sarkar R, Gao GP, Chirmule N, Tazelaar J and Kazazian HH: Partial correction of murine hemophilia A with neo-antigenic murine factor VIII. Hum Gene Ther 11: 881-894, 2000.

44. Qian J, Collins M, Sharpe AH and Hoyer LW: Prevention and treatment of factor VIII inhibitors in murine hemophilia A. Blood 95: 1324-1329, 2000.

45. Fields PA, Kowalczyk DW, Arruda VR, Armstrong E, McCleland ML, Hagstrom JN, Pasi KJ, Ertl HC, Herzog RW and High KA: Role of vector in activation of T cell subsets in immune responses against the secreted transgene product factor IX. Mol Ther 1: 225-235, 2000.

46. Herzog RW, Fields PA, Arruda VR, Brubaker JC, Armstrong E, McClintock D, Bellinger DA, Couto LB, Nichols TC and High KA: Influence of vector dose on factor IX-specific T and $\mathrm{B}$ cell responses in muscle-directed gene therapy. Hum Gene Ther 13: 1281-1291, 2002.

47. Gao G, Lebherz C, Weiner DJ, Grant R, McCullough B, Calcedo R, Bagg A, Zhang Y and Wilson JM: Erythropoietin gene therapy leads to autoimmune anemia in macaques. Blood 103: 3300-3302, 2004. 